\title{
USO DE BANCOS DE TEXTOS LINGÜÍSTICOS PARA LA FORMACIÓN DE TRADUCTORES
}

\author{
Jeanina Umaña Aguilar
}

\begin{abstract}
RESUMEN
En este artículo se detallan las actividades exploratorias en un hipertexto electrónico y el uso de un programa de concordancia como medios eficaces para aumentar la competencia léxica en la lengua fuente. La autora sostiene que este tipo de actividad promueve el desarrollo de tres destrezas específicas que deben incluirse en la formación de los traductores profesionales: conciencia lingüística, actitud reflexiva y uso eficaz de los recursos disponibles.
\end{abstract}

\begin{abstract}
This is a detailed presentation of the author's exploration of an electronic hypertex and use of a concordancer as effective means to increase her lexical competence in the source language. The author holds that this type of activity promotes the development of three specific skills that should be included in the training of proffessional traslators: linguistic awareness, reflection and effective use of resources.

"Los caminos han de ser recorridos y no confundidos con sitios de destino"

Proverbio budista
\end{abstract}

\section{Introducción}

La siguiente tipología de libros que esboza el semiólogo Umberto Eco ejemplifica lo que nos puede motivar a usar bancos de textos lingüísticos e hipertextos electrónicos:

\footnotetext{
Hoy existen dos tipos de libros: aquellos que se leen y aquellos que se consultan. Con los libros para leer se empieza en la página 1, donde, digamos, el autor explica que se ha cometido un crimen. El lector continúa hasta el final, cuando descubre quién es el culpable. Fin del libro y de la experiencia lectora. [...] Las enciclopedias, por supuesto, no están hechas para leerse de corrido. Si quiero saber si fue posible que Napoleón conociera a Kant, tomo los volúmenes K y N [...] y descubro que Napoleón nació en 1769 y murió en 1821, mientras que Kant nació en 1724 y murió en 1804. Es posible que los dos se hayan conocido. Para saberlo con precisión consulto una biografía de Kant. Una biografía de Napoleón, quien conoció a mucha gente, podría obviar un encuentro con Kant; una biografía de Kant, no.

Las computadoras están comenzando a cambiar el proceso de la lectura. Con un hipertexto, por ejemplo, puedo buscar todos los casos en los que el nombre de Napoléon se asocia con Kant. Puedo realizar la labor en unos cuantos segundos (Eco: 2001).
}

Precisamente ese tipo de búsqueda "en unos cuantos segundos" es lo que nos permite hacer un tipo de programa llamado concordancer. Si ingresamos, por ejemplo, al banco 
eléctronico Collins Cobuild o el British National Corpus (BNC), podemos definir qué lexema o frase queremos buscar y el ámbito de la búsqueda, es decir cuántos lexemas antes y después de un elemento dado queremos incluir en el texto. La respuesta es un listado de ejemplos, indexados de modo que posteriormente podemos copiar fragmentos más amplios del texto en que aparece cada ejemplo.

Este tipo de programa, del cual hay una gran variedad, nos permite utilizar un corpus lingüístico extenso que en realidad incluye un número físicamente inmanejable de textos, muchos de ellos escritos para ser leídos y reconfigurados como hipertextos para diversos tipos de referencia, pero que también incorpora transcripciones de textos orales. En este artículo, se ilustra el uso del concordancer en actividades diseñadas para que estudiantes de traducción desarrollen las destrezas específicas de capacidad para la toma de conciencia, capacidad para la reflexión y capacidad para el uso de recursos, detalladas en Umaña (2002) como esenciales en la formación de traductores.

\section{La concordancia en bancos de textos: aprender a traducir mientras se aprende la lengua extranjera}

A inicios del siglo pasado, la traducción como manipulación de formas gramaticales fue un método usado para enseñar lenguas extranjeras como asignaturas del curriculum escolar. Más recientemente, según Eadie (1999), quienes enseñan lenguas extranjeras poco a poco reviven el uso de la traducción para presentar ciertos ítemes de vocabulario, verificar la adquisición de conceptos, etc. La enseñanza de la traducción como área específica se ha centrado tradicionalmente en dar al estudiante oportunidades para ejercitar las destrezas requeridas y retroalimentarlo con la corrección y el análisis del texto meta. Si bien esta es la forma directa de ver la traducción desde una perspectiva didáctica, no es necesariamente la más eficaz, ya que deja totalmente en manos del estudiante la pesada carga de desarrollar las destrezas y estrategias adecuadas. Además, a menudo reduce la retroalimentación a correcciones locales, que en realidad son de poco uso para el desarrollo de la conciencia crítica y de las estrategias transferibles a otro tiempo y otro espacio. A pesar de que la práctica a veces lleva a la perfección, también puede ser fuente de frustración cuando los estudiantes requieren formas específicas de sacarle el mayor provecho al esfuerzo y no se les ofrece ninguna.

Las bases pedagógicas de la enseñanza de la traducción se revelan bastante débiles cuando la metodología subyacente se limita a posibilitar la exposición a textos y dar oportunidades para ejercitar la traducción y corrección, todo probablemente bajo la premisa de que los estudiantes lidiarán con los textos y desarrollarán por sí mismos las destrezas requeridas para producir una "buena" traducción. Sin embargo, podemos imaginar un enfoque de la enseñanza de la traducción en el que el ejercicio directo se complemente con actividades menos directas, pero más explícitas y eficaces, que busquen el desarrollo de la conciencia, la reflexión y la capacidad para el uso de los recursos disponibles, habilidades esenciales que requiere un traductor profesional. Las actividades que impliquen el acceso a bases extensas de textos lingüísticos para el aprendizaje de una lengua, más que como referencia, bien pueden favorecer el desarrollo de las capacidades y estrategias que subyacen esas tres destrezas básicas y, por lo tanto, complementan en forma eficaz la enseñanza "tradicional" de la traducción. 
El término "actividades" quizás no sea el usual para definir el tipo de trabajo que aquí se presenta, por lo cual se define a continuación, para luego analizar sus implicaciones y ejemplificarlo. Comienzo con lo que excluye: estas actividades no son, por varias razones, "tareas" tradicionales (como las de la "instrucción por tareas" o task-based instruction). En primer lugar, estas actividades no se logran a partir de un guión predeterminado ni tienen un resultado predeterminado; por el contrario, el estudiante está a cargo de todo el proceso que incluye planearlas, llevarlas a cabo, evaluar los resultados obtenidos y reportarlos, analizarlos y comentarlos. En segundo lugar, al tratar tanto los métodos como los objetivos como variables controladas por el estudiante, en este enfoque no se aumenta gradualmente la dificultad de las tareas, con lo que se contradice un principio central en la instrucción por tareas. Finalmente, estas actividades son auténticas como pocas otras, pues no duplican ni simulan eventos, ni tampoco dependen del efecto que suponemos tiene el poder dar solución a problemas ficticios para despertar el interés del estudiante. Además, son útiles en sí, o al menos así se espera que las perciban los aprendices.

Dicho brevemente, estas "actividades" sacan provecho de que el aprendizaje a partir de un corpus extenso puede ser estimulante, abierto, intelectualmente retador y divertido y, por lo tanto, no requiere del control estricto ni del estímulo del profesor para ser exitoso, especialmente si los estudiantes tienen interés en la lengua extranjera y su cultura, lo que en principio se debería poder dar por sentado en el caso de estudiantes de traducción que a la vez aprenden la lengua fuente. Al estudiante únicamente se le debe exigir llevar a cabo una investigación de su elección en un banco extenso de textos. Se le debe dejar en claro que puede dedicar todo el tiempo que desee a explorar el texto electrónico y que puede desviarse de la ruta original. Algo fundamental es indicarle que lleve un registro cuidadoso del proceso, con base en el cual pueda escribir un informe sobre las estrategias (tanto las exitosas como las fallidas), los hallazgos (positivos y negativos) y las impresiones personales. Es decir, se le deben dar al estudiante las pautas para que su aprendizaje sea reflexivo.

Se deduce entonces que el "guión" con las instrucciones para las actividades se reduce a un mínimo: se deja al estudiante en absoluta libertad para que tome las siguientes decisiones:

- Selección del estímulo inicial. Esto se refiere a la pregunta, duda o problema que va a contestar o resolver.

- Métodos que usará en la investigación. El estudiante decide sobre las opciones técnicas, como por ejemplo:

- $\quad$ cómo será el diseño de la pregunta

- $\quad$ qué material desea incluir, cómo lo seleccionará, reducirá y contextualizará

- $\quad$ si analiza las concordancias en la pantalla o las imprime

- $\quad$ si trabaja solo o en grupos pequeños

- $\quad$ el número y el tipo de desviaciones, es decir, si se enfoca en fenómenos léxico sintácticos locales, o si más bien lo hace en patrones textuales más generales

- $\quad$ si procede al azar, pasando de una búsqueda a otra, o si investiga un área en forma sistemática, como por ejemplo todos los adjetivos con el sufijo -holic, todos los significados y patrones de la palabra persuasion, etc.

- $\quad$ Forma en que presentará el trabajo final: escrito, oral, esquematizado, impreso, electrónico, etc. 
Conceptualizo estas actividades como viajes de descubrimiento, ya que se caracterizan por la misma naturaleza impredecible y la posibilidad de apertura mental de un viaje, por el carácter imprevisto y sorprendente de muchos encuentros en el camino, así como por los múltiples estímulos para la comunicación que se derivan de cualquier viaje. No importan ni el punto de inicio ni el punto de llegada, pues tan solo suministran mil razones para hacer el viaje. Tampoco importa la ruta que sigamos, ni si llegamos o no al destino que habíamos escogido, siempre y cuando el camino recorrido sea rico como fuente de nuevos conocimientos y experiencias.

Esta forma de ver el aprendizaje a partir de un banco extenso de textos lingüísticos está inspirada en los puntos de vista de Tim Johns (1986, 1991a, 1991b) sobre el aprendizaje como investigación. El punto de partida es dejar que los estudiantes encuentren por sí solos la solución a un problema que conocen o del cual toman conciencia, o la respuesta a una curiosidad o una duda. Sin embargo, deben además desarrollar procedimientos y estrategias que les permitan aprovechar al máximo los recursos con que cuentan (en este caso un extenso banco electrónico de textos lingüisticos), con el fin de llevar a cabo la tarea en forma exitosa y económica. Finalmente, es importante que se sientan libres para explorar fenómenos esperados o no, para desviarse del camino a fin de seguir uno nuevo, o regresar al anterior si llegan al final del nuevo.

Debe quedar claro, entonces, que la finalidad aquí no es la adquisición de un conocimiento o competencia descriptivamente adecuado, si bien este puede ser un resultado valioso, y de hecho probable, en la exploración de la concordancia de un banco extenso de textos. Lo que está en juego, más bien, es el desarrollo de un número de destrezas que se pueden agrupar en la categoría del "saber cómo". En otras palabras, el punto focal está en los procesos más que en los productos, en los métodos más que en los resultados, en la toma de conciencia, la reflexión y la utilización de recursos más que en un concepto tradicional de "lo aprendido".

Con el fin de contestar por qué lo que aprendemos a hacer y, como subproducto, aprendemos, a partir de actividades que incluyan el acceso individualizado a un banco extenso de textos lingüísticos es especialmente relevante para la formación de traductores, presento un ejemplo del tipo de proceso al que me he estado refiriendo, acompañado de los comentarios pertinentes.

\section{Concordancia en un banco extenso de textos: ¿es una de las actividades "de siempre" para el aprendizaje de la lengua?}

Comencé con las preguntas: ¿qué quiere decir "actividades de siempre” en español? ¿Equivalen a las "good old activities" del inglés? Aunque el significado de esta frase puede parecer transparente, deja de serlo si lo deconstruimos en otras preguntas más específicas, tales como:

- ¿ ¿Cuáles palabras se asocian típicamente con la expresión "good old"? Esto no es sino ubicarnos en la conocida perspectiva del significado por distribución y colocación propuesta a mediados del siglo pasado por lingüistas como Firth.

- ¿Qué implicaciones tiene esta expresión con referencia a la intención ilocucionaria del escritor o del hablante?

- ¿Cómo se integra a la textura del discurso (sintaxis, puntuación, etc.)? 
- ¿Está la expresión "de siempre” en relación de antonimia con "buena" o con "mala", dada la existencia en inglés de las expresiones "good old" y "bad old"?

- ¿Cuándo puedo traducir "good old" y "bad old" como "de siempre"?

Planteadas las dudas, pasé a examinar una selección al azar de las expresiones en inglés "good old" y "bad old" en el British National Corpus, del que se puede encontrar una demostración en el sitio www.cobuild.collins.co.uk. Dado que el número total de casos en el corpus es 581, el análisis exhaustivo se torna muy complejo, al menos para los fines de este artículo, que sólo busca ejemplificar la actividad propuesta y analizar sus posibilidades.

El primer hallazgo es que los cotérminos en el rango de una palabra (exceptuando "days") no son simétricos en los textos británicos. "Good old" aparece en forma más consistente con los lexemas "age" ("edad") que, dicho sea de paso, nunca se usa con el sentido de "período histórico" sino más bien en el sentido de "momento en la vida", que, a su vez, no es posible para el posible sinónimo "golden age"; "British" ("británico/a"); "chat" ("conversación"); "days" ("días") y "English" ("inglés"), en tanto la expresión "bad old” aparece con los lexemas “days" ("días"), "habits” ("hábitos") y "ways” ("costumbres").

Las expresiones que se refieren a nacionalidad sólo se dan junto con "good old" y parecen limitarse a los lexemas "England" (Inglaterra) y "English" (inglés) en 14 casos en todo el corpus, así como a "Britain", (Bretaña) y "British/Brit" (británico), en un total de 23 casos. Además, elogian glorias nacionales tales como 'pint' (pinta), 'pub' (taberna), 'ale' (cerveza cruda), 'pork sausages' (salchichas de cerdo) y 'steak and kidney' (estofado con riñones). No obstante, cuando los elementos de la expresión son menos tangibles, la prosodia se torna un tanto negativa, como en los siguientes ejemplos:

Texto H7W (316):
"I see. Without giving me a chance to explain you've decided that not only am I guilty, but I'm a moron as
well." She looked him up and down in open disgust. 'So much for good old British justice!' ("Ya veo. Sin
darme oportunidad de explicar nada, Ud. no sólo decidió que soy culpable, sino además que soy un estú-
pido." Ella lo miró de arriba a abajo con franco disgusto. "Esa es la justicia británica de siempre!").

Texto HR4 (1743):

"... you wouldn't believe me if I said I wasn't drinking of that but you might believe me if I say we'd prefer the good old British way: that nothing happened and there's no blame at all. The psychopath theory can cover the Abbey and for the rest" - she shrugged- 'we can make sure there's no proof' (...Ud. no me creería si le dijera que no estaba bebiendo eso, pero tal vez me crea si le digo que preferiríamos la salida británica de siempre: que nada pasó y que no hay culpa. La teoría del sicópata puede incluir el caso Abbey y en cuanto al resto, dijo encogiéndose de hombros, "podemos asegurarnos de que no haya ninguna prueba...).

En términos generales, me quedó claro que la expresión "good old" parece tener dos usos bien establecidos. Uno es literal, más bien nostálgico, como en "good old traditions" ("las buenas costumbres de antaño"), "good old black and white" ("en el blanco y negro de siempre") o incluso a veces nacionalista, como en

\footnotetext{
'...good old England. Up the empire'; 'indicating altitudes in foreign metres [...] and abandoning the good old British feet' ("la Inglaterra de siempre. Que viva el imperio, que ahora marca la altitud en metros extranjeros $[. .$.$] y abandona el tradicional pie británico").$
} 
El otro significado juega con el primero para mofarse de la actitud subyacente, como en

'...good old Britain: not only doing the wrong thing, but unable to keep it secret' ("la Gran Bretaña de siempre: que no solo hace lo incorrecto sino que es incapaz de mantenerlo en secreto"),

o para atacarla abiertamente como en

\begin{abstract}
Texto ACP (537)
Today's society, the adverts pointed out, is too knowing, too soiled, and too corrupt -as if Fifties' America was some kind of zenith in Western civilisation. Yes, the good old US Fifties, where trade unionists got shot dead for striking in Detroit, where blacks got lynched by the Klan without hindrance in the South, and where Senator Joe McCarthy or FBI boss J. Edgar Hoover destroyed peoples' lives for having beliefs contrary to their own. (La sociedad actual, acotaban los oponentes, sabe demasiado, es demasiado sucia y demasiado corrupta. Como si la década de los 50 en los Estados Unidos fuera algo así como la cima de la civilización occidental. Sí, los gloriosos años cincuenta en los Estados Unidos, donde mataban a los sindicalistas por hacer huelgas en Detroit, donde los negros eran linchados impunemente por el Klan en el Sur y donde el Senador Joe McCarthy o el Director del FBI J. Edgar Hoover le arruinaban la vida a la gente por tener creencias contrarias).
\end{abstract}

Lo que parece ser especialmente relevante desde la perspectiva del aprendizaje es que cuando la intención del autor no es transmitir un tono nostálgico, esto normalmente se indica en inglés mediante el uso de la puntuación -a menudo mediante el uso de comillas invertidaso mediante una explicación o una expansión que guía la interpretación, como en el ejemplo de 'U.S. fifties'. Esto se comprende cuando en los textos la década de los 50 aparece como el prototipo de "good old days" o "los buenos tiempos" en la cultura británica, junto con las décadas de los años 20, 30 y 40, según lo aclara uno de los ejemplos:

\title{
Texto K97 (14085):
}

When people talk about the good old days they usually mean the 1920s to the early 1950s. (Cuando la gente habla de los buenos tiempos, por lo general se refiere al período desde la década de 1920 hasta principios de los años 50).

Esto se ilustra también al comparar el texto anterior con otro ejemplo, tomado de una transcripción:

\section{Texto HHV (12720):}

I am afraid that the Labour party is still in the 1960s and 1970s. I very much hope that we shall never see a return to those bad old days. (Me temo que el Partido Laborista todavía está en los años 60 y 70 . Realmente espero que nunca regresemos a esos malos tiempos).

Resulta interesante, además, que tanto "good old days" como "bad old days" parecen usarse muy raras veces para describir experiencias de vida personal. Por lo general, se refieren a una experiencia del pasado compartida y tienen entonces una fuerte función sociológica. "Good old days" se refiere al tiempo en que la vida era fácil, antes de que los jóvenes se nos fueran de las manos, una época de conducta decente, y "bad old days" se refiere a una época anterior a la alta tecnología, de crisis y antagonismos de clase, cuando 'los patronos estaban muy ocupados contratando gente para que presionara a otros y se reportara con inspectores que no tenían nada que ver con la capacitación y que no producían resultados.' [J9L (119)]

Regresando a las preguntas planteadas al inicio de esta sección, podemos ahora ver que un enunciado sobre el significado (que utilizo como sinónimo de 'uso') de estas frases en 
inglés y en español no es tan directo como podríamos haber pensado si lo hubiéramos visto tan solo desde la perspectiva intratextual.

Una vez contestada la primera pregunta, paso a analizar algunas de las numerosas disgresiones hechas en el curso de las búsquedas sugeridas por los textos mismos. En primer lugar, decidí hacer búsquedas para estudiar las reglas de los elementos cotérminos con las frases "rose-tinted" ("color de rosa") y "golden age" ("edad dorada"), las cuales se habían presentado en el siguiente contexto:

\footnotetext{
Texto CCE (660):

Hankering after the 'good old days' is a way of always viewing history with rose-tinted glasses. Looking backwards with either envy or merely admiration tends to distort history. It has already happened with Victorian England, for example. No longer is it to be seen as an age of poverty, disease and false hopes. Rather it assumes the image of a golden age of progress and stability. (Añorar los buenos tiempos es una forma de ver siempre la historia con lentes color de rosa. Mirar atrás ya sea con envidia o simplemente con admiración tiende a distorsionar la historia. Ya sucedió con la Inglaterra victoriana, por ejemplo, que ya no ha de ser vista como una época de pobreza, enfermedad y falsas espectativas sino que, por el contrario, adquiere la imagen de una edad dorada de progreso y estabilidad).
}

La expresión "rose-tinted" resultó ser de uso bastante frecuente en el sentido figurativo antes ilustrado, junto a lexemas como "spectacles" ("espejuelos", 8 casos), "picture" ("imagen", 2 casos), "retrospection" (retrospección, 1 caso), "vision” (visión, 1 caso). Por otra parte, si bien la palabra "spectacles" aparece siempre en sentido figurativo, no sucede lo mismo con "lenses" ("lentes", 3 casos) que mantiene su sentido literal, en tanto que "glasses" ("anteojos") puede usarse o no en sentido figurativo (1 ejemplo para cada caso).

Pasé luego a buscar casos de la palabra "tinted" precedida de varios colores ("browntinted", "green-tinted", "purple-tinted", "blue-tinted", "yellow-tinted", "red-tinted", etc.), y encontré que únicamente el color verde se usa figurativamente en esta expresión, cuya traducción es a menudo figurativa:

Texto A0G (39):

Organic advice: Another batch of green-tinted tips from the experts at the National Centre (Consejo orgánico: Otro conjunto de consejos ecológicos de los expertos del National Centre)

\section{Texto HH3 (371):}

... big business is to promote consumption. So however green-tinted companies become... (...hacer negocio en grande es promover el consumo. De modo que no importa cuán ecológicas se vuelvan las empresas...)

Pasé luego a examinar lo encontrado para "golden age" ("edad dorada"). Aunque podría argumentarse que la frase se puede ver como sinónimo de "good old days" (ambas, por razones para nada obvias, ocurrieron antes y no después de algo, y ambas se usan como idealizaciones del pasado) los lexemas cotérminos con "golden age" sugieren que en los textos británicos la expresión tiene menos relación con Gran Bretaña o Inglaterra (la búsqueda en un rango de 2 palabras dio: 1 caso de "British", 1 de "England", 2 de "English"). Además, señalan una actitud mucho más cosmopolita, pues el mismo rango de dos palabras dio como resultado un caso para cada uno de los siguientes lexemas: Wales, Dutch, European, Chinese, German, Australian, Japanese, Scottish, Ireland (galés, holandés, europeo, chino, alemán, australiano, japonés, escocés, Irlanda). De igual forma, no sugieren burla ni antagonismo, aún en casos en que critican el concepto en vez de apoyarlo, como en: 


\section{Texto CE1 (1258):}

Whilst not wishing to create visions of the past as a 'Golden Age' (it was certainly not), it cannot be denied that... (Si bien no se desea crear una imagen del pasado como una "Edad Dorada" (ciertamente no lo fue), no se puede negar que...)

Con frecuencia se indica cuál es la "edad dorada" que se describe: la de los caricaturistas, la de los profesionales, la de los libros infantiles, etc., con la nota adicional de que la preposición "for" más la especificación de cuál, nunca aparece en posición inmediatamente posterior a "good old days" ni a "bad old days". Dichas observaciones parecen sugerir que "golden age" podría tener una menor connotación sociológica y cultural que "good old days", y que las implicaciones de cada una con relación a la intención y al punto de vista de un autor son diferentes.

La expresión "not be (such) a bad old stick" en un texto producto de la búsqueda sobre "golden age" me llevó a hacer búsquedas para "old stick(s)" y posteriormente para "stick/sticks" como sustantivos (después de un intento desafortunado en el que no se especificó la clase de palabra, lo que produjo todos los casos de "stick" y "sticks" en el corpus, un número inmanejable). Resultó que "old stick" es por lo general una persona, mientras que "old sticks" también pueden ser cañas de pescar o palitos. La expresión a veces parece indicar un tono afectuoso, aunque probablemente un tanto condescendiente (como en la forma negativa "not a bad old stick" o en "funny old stick"), y otras veces expresa un juicio claramente negativo, especialmente cuando se refuerza con "dry" o "dried-up", aclarado aún más por el contexto, como en:

\section{Texto GUD (21):}

She's a dried-up old stick now, Andy. Sings in the Methodist choir. I can't believe my memory when I look at her. (Ahora es una vieja enjuta, Andy. Canta en el coro metodista. Cuando la veo no puedo creer lo que ha cambiado).

\section{Texto K5A (4129):}

An apparently dry old stick, secure in the fraternity of the common room, and content with the comradeship of his brother, Lewis is approaching 60 when he learns to love a woman... (Aparentemente viejo, cómodo en la fraternidad de la habitación compartida y satisfecho con la camaradería de su hermano, Lewis se acerca a los 60 cuando aprende a amar a una mujer...)

Los sustantivos "stick" y "sticks" resultaron ser sumamente abundantes en usos figurativos y son fuente de un número interesante de usos específicos, tales como 'stick of celery' (rama de apio), 'coctail stick' (palillo de dientes), 'golf stick' (palo de golf), 'stick of French bread' (bollo de pan francés o baguette), 'stick of furniture' (mueble), 'cinnamon stick' (astilla de canela). También, resultó que ni 'a big stick' ni 'the sticks' son por lo general trozos de madera. El primero se refiere por lo general a algún tipo de imposición (recuérdese, por ejemplo, la política estadounidense del Big Stick), o trato desigual, como en los siguientes dos casos:

\section{Texto HGM (3205):}

In your shoes I'd have expected him to come prepared with a big stick. I "You mean I should have guessed he'd come prepared to blackmail us?" she enquired (Si fuera Ud., yo habría esperado que él viniera preparado para imponer su punto de vista... "Ud. quiere decir que debí haber supuesto que vendría preparado para extorsionarnos?" preguntó ella). 


\section{Texto ECH (115):}

He was annoyed, he said, that deer forest managers were given the kid glove treatment while hillwalkers and climbers had the big stick of stiffer sanctions waved at them (Estaba sorprendido, dijo, de que a los administradores de los bosques donde habitan los venados se les tratara con guantes de seda mientras que a los caminantes y practicantes del montañismo se les imponía sanciones más severas).

Por otra parte, "the sticks" se refiere a un lugar rural, descrito en términos no precisamente afectuosos:

\section{Texto HTL (2662):}

As we approached the Mercedes, Bernice said: "Was that a friend of yours? Do you want to ask him along?" "Never seen him before," I said. I Some of the people I know regard Hackney as the sticks and half expect to see herds of grazing wildebeest when they come to visit me. (Conforme nos acercábamos al Mercedes, Bernice dijo: "¿Ese era tu amigo? ¿Quieres pedirle que nos acompañe?" "Nunca antes lo había visto", repliqué. I Algunas personas que conozco creen que Hackney es muy rural y casi que esperan ver rebaños de animales salvajes pastando cuando vienen a visitarme).

Por último, recordé que la expresión "the stick and the carrot" había sido de especial dificultad en un texto analizado por los estudiantes de un curso de traducción, por lo cual me pareció que merecía ser investigada. La búsqueda de "stick(s) [noun] and carrot(s)" en ese orden y a la inversa y con un rango de diez palabras, dio como resultado 40 casos, la mayoría de ellos relevantes, excluyendo unos cuantos sobre recetas de cocina con zanahoria y apio. La frase resultó ser muy productiva, tanto en lo léxico como en lo sintáctico, y ejemplos como los siguientes son ricos en glosas explicativas:

\footnotetext{
Texto B2F (1422):

The answer is to use punishment sparingly, very much as a last resort. Another useful way to use punishment is in combination with positive reinforcement. This is the carrot and the stick approach, the carrot being positive reinforcement and the stick punishment (La respuesta es usar el castigo con medida, como último recurso. Otra forma útil de usar el castigo es en combinación con el refuerzo positivo. Este es el enfoque del palo y la zanahoria, en el que la zanahoria es el refuerzo positivo y el palo el castigo).
}

\section{Texto HGP (2290):}

Because shareholders know all this, they try to give managers a direct incentive to care about profits and cost reduction. Senior executives get large profit-related bonuses. These are a carrot, for those who like carrots; but shareholders have no accompanying stick, which effective two-handed discipline would require. (Como los accionistas saben todo esto, tratan de darle a los gerentes un incentivo directo para que se preocupen por las ganancias y la reducción de costos. Los ejecutivos de más rango obtienen cuantiosos reconocimientos relacionados con las ganancias. Estos son una zanahoria, para los que les gusta la zanahoria; mas para los accionistas no existe la vara del castigo que exigiría la disciplina eficaz y justa).

Seis ejemplos de la frase anterior venían de un mismo texto (CEF, Status: what it is and how to achieve it). Los ejemplos llamaron mi atención, por lo que procedí a examinar más de cerca el contexto inmediato y noté que la palabra "power" (poder) y sus derivados constituyen palabras claves de este texto. En consecuencia, hice otras búsquedas para la palabra "power" en el corpus, así como en este texto en particular. En el corpus, la palabra "power" a menudo parece referirse a una imposición de algún tipo y parece tener una connotación más o menos negativa, de la que carece, por ejemplo, el adjetivo "powerful" (poderoso). Por otra parte, en el texto examinado, la palabra tiene una connotación muy positiva, expresada en forma explícita: 


\begin{abstract}
This chapter examines different ways of exercising power. It gives practical suggestions on how to develop your influencing skills, on how to become more versatile when influencing and on how to choose what tactics are best for the situation. [...] Power gives us the ability to control, to choose and to assert our independence. [...] Knowledge and skills are a valuable source of power that we can build on and develop. (Este capítulo examina diferentes formas de ejercer el poder. Da sugerencias prácticas sobre cómo desarrollar sus destrezas influyentes, cómo llegar a ser más versátil para influir y cómo seleccionar cuáles son las mejores tácticas para una situación dada. [...] El poder nos da la capacidad de controlar, escoger y afirmar nuestra independencia. [...] El conocimiento y las destrezas son una fuente valiosa de poder a partir de la cual podemos desarrollarnos y crecer).
\end{abstract}

La lectura de la totalidad de este texto motivó nuevas búsquedas: la frase 'the power behind the throne' (el poder detrás del trono), que a su vez dio lugar a búsquedas sobre "behind the" y "power(s) behind". Las respuestas indican que la frase está de hecho lexicalizada (12 casos), con variantes tales como 'the power behind the gods' (el poder detrás de los dioses) y 'the power behind the dictator' (el poder detrás del dictador). Mientras examinaba el resultado para la búsqueda de "behind the" (detrás de), noté las siguientes frases, que constituyen excelentes ejemplos de cotérminos retadores para el traductor (Después de cada término se indica la frecuencia entre paréntesis):

"work (22) behind the scenes"

"driving main (5) force(s)"

"moving (4) pressure(s) behind"

"motivating (10) / general (3) / guiding (2) / fundamental (2) principle(s) behind"

Es interesante que esta última expresión ("the principle behind") se use más a menudo en posición de tema y no de rema, y parece funcionar como señal para el lector de que según el autor la información que sigue es realmente relevante:

Texto J3H (528):

The principle behind substitution is that it should carry no environmental penalty (El principio que subyace la sustitución es que no debería acarrear castigo ambiental).

Texto EA4 (357):

The principle behind care management is to evolve quality systems based on users "needs" (El principio que sustenta la administración de la atención es desarrollar sistemas de calidad basados en las necesidades de los usuarios).

Texto K5H (282):

The principle behind the act is that marriage should be regarded as a partnership which is dissolved on divorce allowing both parties to have a 'clean break' (El principio que sustenta el acto es que el matrimonio debe verse como una sociedad que se disuelve con el divorcio, lo que permite que ambas partes tengan una 'separación decente').

La expresión "We power dressed in the 1980s" me hizo buscar formas de "dress" y formas de "power" en un ámbito de diez palabras. Obtuve catorce casos de "power dressing" y dos de "power dressed", lo cual probablemente indica una frase lexicalizada, con fuerte carga cultural, como en:

Texto CEF (587):

When we power dressed we made our bodies look angular and more aggressive, with hard, sharp lines. (Cuando nos vestimos para demostrar poder, hicimos que nuestros cuerpos se vieran angulares y más agresivos, con líneas duras y bien definidas). 


\begin{abstract}
Texto K2U (483):
NOTHING characterised the late 1980s so much as the short skirt.

Welcomed by women of almost all ages as comfortable and a declaration of female sexuality, the short skirt suit with power dressing shoulder-padded jacket was simply the best for oozing confidence at the office. (Nada caracterizó los últimos años de la década de 1980 como la minifalda. Bien recibida por mujeres de casi todas las edades como una prenda cómoda y una declaración de sexualidad femenina, el traje de minifalda y saco con hombreras que confería poder era simplemente lo mejor para sentir una gran confianza en la oficina).
\end{abstract}

En este punto pude haber hecho búsquedas para "1980s", "80s" y "eighties", y posiblemente para todas las variantes de "70s", "60s", "50s", "40s", etc., revisando los términos que aparecen en cada caso. De hecho inicié la búsqueda, pero pronto me di cuenta de lo numerosas que eran las respuestas y decidí retomar esta búsqueda en un viaje posterior.

\title{
3. La concordancia en bancos extensos de textos lingüísticos y la educación de traductores conscientes, reflexivos y con abundantes recursos
}

Si bien anteriormente afirmé que esta actividad debería verse como un ejemplo (que responde a mis propios intereses personales y mis propias estrategias de aprendizaje), más que como un prototipo (un viaje "típico") del enfoque que estoy tratando de describir, espero haber aclarado cuál es el tipo de actividad que tengo en mente para los estudiantes. Veamos a continuación la relación de la actividad con tareas definidas (Umaña 2003) como prioritarias para la educación de traductores que a la vez son aprendices de la lengua fuente.

\subsection{Sobre la toma de conciencia}

En vista de que el análisis de un banco extenso de textos pone en un primer plano los supuestos culturales que subyacen el uso lingüístico (como en el caso de "good old/bad old"), puede ayudarle al traductor a superar no sólo la limitación de la falta de intuición en la lengua extranjera, sino que además, paradójicamente, puede ayudarle a superar la limitación, señalada por Venuti (1992), de la intuición en la lengua materna. Esta intuición a menudo contribuye a ocultarle al hablante la ideología que la lengua materna transmite y de la cual corre el riesgo de ser un emisario inconsciente o involuntario. El uso de un banco extenso de textos nos obliga a sospechar de la transparencia.

Una vez que los estudiantes entienden lo que pueden hacer con un corpus extenso, dejan de contentarse con soluciones de traducción aproximada, tratan de encontrar lo que está más allá de las palabras de los textos que traducen y deconstruyen no sólo el significado sino también la intención que transmiten (y que podría incluso oponerse a lo que el autor está tratando de hacernos creer que está haciendo, como bien lo explica Louw 1993). Las actividades con corpus extensos nos enseñan a hacernos preguntas al mismo tiempo que nos enseñan a ver posibles respuestas; es decir, nos enseñan a notar, generalizar, plantear hipótesis y refinarlas, sacar conclusiones, etc. Esto se logra tanto al centrar nuestra atención en observaciones intratextuales, como al notar patrones léxicos (como en el texto anterior sobre estatus) y como en las observaciones intertextuales, por ejemplo, al trabajar con la lexicalización y los supuestos 
culturales. En este sentido, las búsquedas parecen constituir medios para ejercitar y refinar un conjunto de instrumentos cognitivos que difícilmente se pueden desarrollar mediante la atención intratextual en un solo texto, tan común en las tareas tradicionales de traducción directa.

\subsection{Capacidad para la reflexión}

Con relación al desarrollo de algunas de las destrezas cognitivas que se requieren específicamente para traducir, pareciera que el trabajo con bancos extensos de textos constituye, en primer lugar, un medio para aumentar la competencia léxica que, desde la perspectiva actual en lingüística aplicada, constituye una "destreza" más que un inventario de nociones y requiere contacto con la expresión auténtica del lenguaje. Como bien señalan Carter y McCarthy, "es imposible enseñarle a los estudiantes todas las palabras que necesitan saber, y por eso es importante enseñarles estrategias para adivinar significados que les permitan abordar palabras desconocidas y perder la dependencia del diccionario" (1988: 42, traducción mía; ver también Robinson 1989)

Cabe aquí recalcar que parte de la tarea que enfrentan los profesores de lenguas extranjeras es crear condiciones que compensen realmente la falta de contacto del estudiante con la lengua que están aprendiendo, sobre todo la falta de contacto "con aquellos significados establecidos y con las combinaciones fijas de palabras cuya recurrencia caracteriza la actuación léxica cotidiana, a la vez que evitan recurrir a técnicas de memorización estériles y desacreditadas" (Cowie 1988, 1992).

En segundo lugar, el trabajo con bancos extensos de textos es un medio para desarrollar la habilidad para 'notar, darse cuenta', la cual, según algunos investigadores, es crucial para aprender. Además, este tipo de trabajo es un medio para refinar y traer al nivel consciente las destrezas cognitivas requeridas para la negociación del significado según las enumeran Beaugrande y Dressler (1981), para quienes la comunicación textual requiere capacidad para:

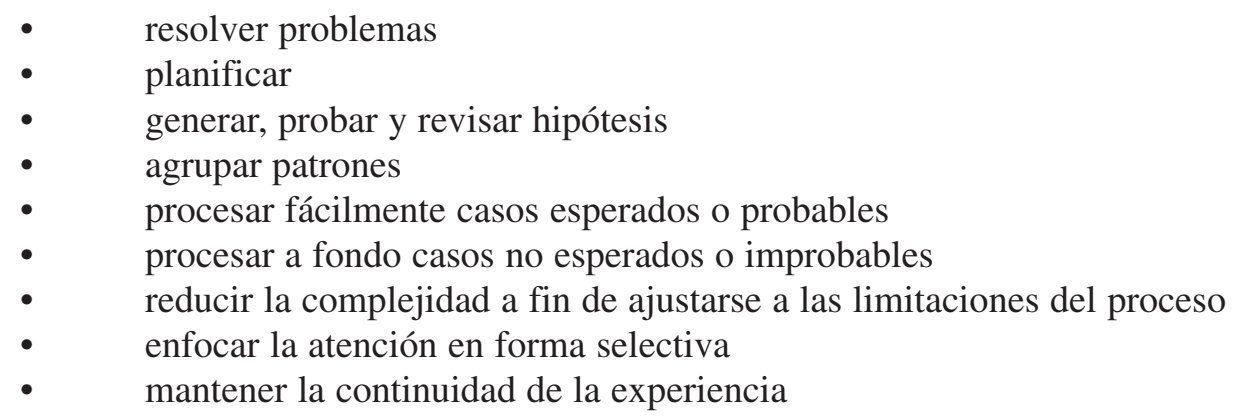

En la medida en que el análisis textual en bancos extensos obliga al estudiante a observar, inferir y generalizar, es conducente a la reflexión, una habilidad que parece ser fácilmente exportable y especialmente valiosa en vista de la "apuesta a la traducción" de la que depende el oficio según Eco. Cuando la reflexión se acompaña con la conciencia, disminuye el riesgo 
de la sumisión. Según Widdowson (1991), un enfoque de la pedagogía de lenguas basado en la actuación, tal como el que ofrece la linguíistica textual, corre el riesgo de obstaculizar la libertad del estudiante, forzándolo a someterse a hábitos culturales que bien podrían contraponerse a los suyos propios. Sin embargo, este riesgo parece referirse únicamente a aquellas situaciones en que el estudiante no está equipado con los instrumentos necesarios para hacer una elección racional entre la sumisión y la afirmación según el problema que enfrenta y de acuerdo a su propio "proyecto de traducción”, según lo define Simon (1996).

En otras palabras, el conocimiento de lo que típicamente se dice o no, se escribe o no en una cierta comunidad lingüística es un componente fundamental de la competencia de un traductor, siempre que vaya acompañado de la capacidad para interpretar textos y tareas exacta y críticamente. Las actividades como las aquí descritas, basadas en el trabajo con un banco extenso de textos, parecen suministrar esa competencia y esa capacidad.

Finalmente, es probable que la observación cuidadosa de las estrategias adoptadas para la comunicación textual exitosa tenga efecto, tanto en el procesamiento del texto meta como en el del texto fuente. Por ejemplo, el poder observar que la frase "the principle behind" a menudo se usa para subrayar información relevante podría estimular la reflexión sobre las estrategias personales de escritura y sugerir, además, formas de mejorarlas.

\subsection{La capacidad para el uso de recursos}

Por último, las actividades de aprendizaje basadas en bancos electrónicos de textos constituyen oportunidades para el aprendizaje autónomo, ya que educan al estudiante para:

(a) resolver problemas reales con recursos disponibles;

(b) explotar su competencia y capacidad para hacerle frente a exigencias retadoras, aumentando así, un poco cada vez, la competencia y la capacidad;

(c) desarrollar las destrezas técnicas específicas que requiere el uso de un banco extenso de textos y que son necesarias para un tipo de navegación electrónica más sofisticada;

(d) saber dónde encontrar solución a los problemas que se pueden presentar en el curso de una tarea de traducción. Los estudiantes necesitan ser educados en el uso de bancos electrónicos para que los aprovechen con fines de referencia y para evitar así el riesgo que conlleva una actitud acrítica del tipo expresado en "está en el corpus (por ejemplo, un diccionario); por lo tanto, es correcto" o "no está en el corpus; por lo tanto, es incorrecto".

El trabajo con bancos electrónicos, por lo tanto, propicia una mayor autonomía, confianza en sí mismo y flexibilidad. En resumen, conduce a saber utilizar los recursos sobre los que se sustenta la adquisición de la experiencia profesional. Es precisamente esta capacidad, junto con la reflexión y la conciencia, lo que creo que debemos tratar de desarrollar en la educación de los traductores, al ser estas las características que distinguen a un Traductor, así, con mayúscula, de los aspirantes sin destrezas que constituyen una herencia lamentable de los tiempos malhadados, como bien se dice en este extracto del BNC:

\section{Texto ECG (1461):}

The quality of translation here, with its simpering rhythms and pathetic language, proves that in this matter the major publishers have moved on hardly at all from the bad old days when the employment of unskilled 


\begin{abstract}
amateurs or stilted romantics in translation work marred the English publication of some of the great accounts of European mountaineering. (Aquí la calidad de la traducción, de ritmo afectado y patético lenguaje, prueba que las principales casas editoriales casi no han avanzado en este tema desde aquellos tiempos malhadados en que, debido al empleo de aficionados sin destreza alguna o de traductores pomposos, se desfiguraron las publicaciones en inglés de varias de las grandes narraciones del montañismo europeo).
\end{abstract}

\title{
4. Conclusiones
}

Una de las razones por las cuales la enseñanza de la traducción, que por lo general se entiende como ejercicio y corrección, se percibe a menudo como ineficaz y tentativa, es que todavía carece de un sólido respaldo pedagógico. No será sino hasta que nos demos cuenta de que poder traducir y poder enseñar a traducir no son la misma cosa, que la enseñanza de la traducción seguirá siendo en general una actividad de capacitación proclive a mantenerse a bajo nivel, carente de conciencia crítica en cuanto a sus propios fines y métodos y, en consecuencia, incapaz de llenar las espectativas de los estudiantes ni los requisitos del mundo profesional.

En este artículo, sugiero que el aprendizaje de la traducción se puede beneficiar mucho más del desarrollo de categorías amplias de destrezas que de la adquisición de competencia en dominios específicos del saber, tales como la ingeniería o la economía. Estas destrezas, llamadas aquí conciencia, reflexión y capacidad para el uso de recursos, no parecen desarrollarse eficazmente sólo mediante el ejercicio. Por el contrario, requieren atención específica a los aspectos intratextuales e intertextuales del uso lingüístico, la exigencia explícita de reflexionar y notar, así como el estímulo explícito para utilizar los recursos disponibles en la solución de problemas retadores. He tratado de ilustrar una forma en la que el acceso personal y abierto a los extensos bancos electrónicos suministra esa atención, esa exigencia y ese estímulo.

$\mathrm{Si}$ a estas consideraciones agregamos que ahora el acceso a importantes bancos de textos como el BNC o The Bank of English es relativamente fácil, y sin duda lo serán aún más en el futuro, que su uso requiere relativamente poco esfuerzo y habilidad técnica y que la carga para el profesor, en términos de capacitación técnica y supervisión, es relativamente liviana, podemos afirmar que el acceso personal y abierto a los bancos extensos de textos lingüísticos como un medio para el aprendizaje, más que como acceso a sitios de referencia, puede ser una oportunidad valiosa y viable para complementar las actividades tradicionales de traducción y enriquecer un entorno de aprendizaje que con urgencia requiere mejorar.

\section{Bibliografía}

Alatis, J. E. (Comp.). 1991. Georgetown University Round Table on Language and Linguistics. Washington D.C.: Georgetown University Press.

Arnaud, P. J. y H. B. Joint. (Comps.) 1992. Vocabulary and applied linguistics. London: Macmillan.

Baker, M., G. Francis y E. Tognini Bonelli. (Comp.) 1993. Text and technology. Filadelfia y Amsterdan: John Bejamins. 
Beaugrande, R. A. de y W. U. Dressler. 1981. Introduction to text linguistics. London and New York: Longman.

Carter, R. 1993. "Language awareness and language learning”. En Hoey, M. (comp.).

Carter, R. y M. McCarthy. 1988. “Developments in the teaching of vocabulary”. En Carter, R. y M. McCarthy (Comps.).

(Comps.). 1988. Vocabulary and language teaching. London: Longman.

Cowie, A.P. 1988. "Stable and creative aspects of vocabulary use”. En Carter, R. y M. McCarthy (Comp.).

Cowie, A.P. 1992. "Multiword lexical units and communicative language teaching". En Arnaud, P.J. L. y H. B. Joint (Comp.).

Eco, U. 1995. "Riflessioni teorico-pratiche sulla traduzione”. En Neergard, S. (Comp.).

2001. "La imaginación virtual". Semanario Universidad. No. 1425. 16 de marzo.

Eadie, Jacqueline. 1999. A Translation Technique. English Teaching Forum. Vol. 37, No. 1.

Gavioli, L. 1996. "Corpora and the concordancer in learning ESP: an experiment in a course of interpreters and translators". Ponencia presentada en el $18^{\circ}$ Congreso de la Associazione Italiana di Anglistica. Génova: 30/9 al 2/10.

Hoey, M. 1991. Patterns of lexis in text. Oxford: Oxford University Press.

Hoey, M. (Comp.). 1993. Data, description, discourse. London: Harper Collins.

Johns, T. 1986. “Micro-concord: A language learner's research tool”. System. 14 (2): 151-62.

1991a. "Should you be persuaded? Two samples of data-driven learning materials". En Johns T. y P. King (Comp.).

1991b. "From printout to handout: grammar and vocabulary teaching in the context of data-driven learning". En Johns T. y P. King (Comp.).

Johns T. y P. King (Comp.). 1991. Classroom concordancing. ELR Journal. 4. Birmingham: University of Birmingham.

Louw, B. 1993. “The diagnostic potential of semantic prosodies”. En Baker, M., G. Francis y E. Tognini-Bonelli (Comps.). 
Neergard, S. (Comp.) 1995. Teorie contemporanee della traduzione. Milan: Bompiani.

Robinson, P. J. 1989. “A rich view of lexical competence”. ELT Journal. 43 (4): 274-81.

Simon, S. 1996. Gender in translation. Londres y Nueva York: Routledge.

Stubbs, M. 1996. Text and corpus analysis. Oxford: Blackwell.

Umaña, Jeanina. 2003. "Relación entre la enseñanza de lenguas y la enseñanza de la traducción". Publicación pendiente. Kánina. Universidad de Costa Rica.

Venuti, L. 1992. "Introduction”. En Venuti, L. (Comp.). Rethinking translation. Londres y Nueva York: Routledge.

Widdowson, H.G. 1983. Learning purpose and language use. Oxford: Oxford University Press.

1984. Explorations in applied linguistics 2. Oxford: Oxford University Press.

1991. "The description and prescription of language". En Alatis, J. E. (Comp.). 